\title{
Scheub, Harold. -- Story. Madison, University of Wisconsin Press, 1998, 351 p.
}

François-Xavier Fauvelle-Aymar

\section{(2) OpenEdition}

1 Journals

Édition électronique

URL : http://journals.openedition.org/etudesafricaines/74

DOI : 10.4000 /etudesafricaines.74

ISSN : $1777-5353$

Éditeur

Éditions de l'EHESS

Édition imprimée

Date de publication : 1 janvier 2001

ISBN : 978-2-7132-1385-4

ISSN : 0008-0055

\section{Référence électronique}

François-Xavier Fauvelle-Aymar, « Scheub, Harold. -- Story. Madison, University of Wisconsin Press, 1998, 351 p. », Cahiers d'études africaines [En ligne], 161 | 2001, mis en ligne le 30 avril 2003, consulté le 20 avril 2019. URL : http://journals.openedition.org/etudesafricaines/74 ; DOI : 10.4000/

etudesafricaines.74

Ce document a été généré automatiquement le 20 avril 2019

(c) Cahiers d'Études africaines 


\title{
Scheub, Harold. -- Story. Madison, University of Wisconsin Press, 1998,
} $351 \mathrm{p}$.

\author{
François-Xavier Fauvelle-Aymar
}

Cet ouvrage au titre laconique (et sans sous-titre) se présente comme une étude sur l'essence du conte (story) et de l'art de raconter (storytelling), étude qui se trouve être appliquée à la littérature orale sud-africaine. C'est donc un essai qui s'inscrit à la fois dans le registre de la théorie littéraire et dans celui de l'africanisme. L'argument principal est avancé dès la première page de l'introduction : s'opposant en bloc aux théories du conte qui ne s'intéressent qu'à son « message » moral (ignorant par là même son « vrai » message) ou encore à sa structure (ignorant par là même son « vrai » pouvoir sur les auditeurs), l'auteur veut défendre l'idée que l'intérêt réel des contes réside dans un contenu émotionnel à plusieurs strates savamment arrangées par le conteur (ou plutôt par la conteuse, la plupart des personnes mises ici à contribution pour leur talent narratif étant des femmes). À partir de là, le livre se déploie de façon kantienne en deux parties dont l'une comprend les éléments de cette nouvelle théorie des émotions (partie intitulée " First principles »), l'autre une explicitation de leurs modes de combinaison (partie intitulée « Palimpsest »). À chaque étape, les contes sud-africains servent d'illustrations. Le résultat est décevant et irritant. D'abord en raison du ton de l'auteur qui, tout au long de ces pages, ne semble procéder que par enchaînements de définitions plus ou moins abstruses qui laissent le lecteur dans la position de l'étudiant contraint d'apprendre par coeur les doctes conclusions d'un professeur d'herméneutique avant d'être autorisé à passer à l'étude des textes proprement dits. À cela s'ajoutent les hypothèses présentées comme des certitudes avérées (telle cette idée maintes fois répétée selon laquelle les peintures rupestres des Bushmen ne seraient que des contes visuels) ou encore les arguments d'autorité qui laissent flotter dans cet ouvrage un désagréable parfum de science exacte. Ainsi apprend-on que les éléments du conte sont au nombre de quatre (pas un de plus, pas un de moins) : l'image, la narration (narrative), le rythme, et le trope. La première partie se découpe d'ailleurs en autant de chapitres, chacun d'eux étant consacré à l'un de ces éléments, récits à l'appui. Mais outre que l'on ne voit pas en quoi 
chacun des contes proposés peut être une illustration adéquate de l'élément en question (surtout à supposer que le conte « réussi ", selon l'auteur lui-même, soit celui qui mêle adroitement les quatre éléments), tous sont suivis d'un commentaire d'autant plus surprenant qu'il semble se détourner des axiomes initiaux pour fournir une interprétation des plus banales.

Isolés dans la première partie, les éléments se réconcilient dans la seconde qui présente un profil différent. Là sont présentés successivement trois conteurs, et donc trois histoires, chacune précédée de son doppelgänger, à savoir l'histoire passée ou présente « that haunt[s] and give[s] context to the story being performed» (p. 185). Cette reconstitution hypothétique de palimpsestes oraux semble assez convaincante dans le cas de //Kabbo, un Bushman qui fut l'informateur du linguiste et folkloriste W. H. I. Bleek à la fin du XIX siècle $^{1}$ (le conte ici utilisé, « Mantis and the All-devourer », est d'ailleurs une reprise de celui publié par Bleek) : //Kabbo peut en effet fort bien avoir entendu raconter l'histoire rapportée à Bleek par un autre Bushman, Diä !kwain, et qu'on peut apercevoir en trame de son propre récit. Moins convaincant en revanche est le cas où le doppelgänger d'un conte xhosa est une histoire swazi, dont Scheub nous dit simplement qu'elle est très répandue en Afrique australe. Mais pas du tout convaincant est l'exemple de Pauline Smith, écrivain britannique qui consacra plusieurs récits à l'Afrique du Sud profonde, celle des Afrikaners du Karoo, et dont l'histoire ici présentée (seul exemple d'histoire écrite utilisée dans l'ouvrage) offrirait des résonances avec un conte xhosa. Pourquoi pas? Mais il faudrait, pour commencer la démonstration, plus qu'une simple supposition, ou bien, si c'est d'un phénomène culturel plus général qu'il s'agit, traquer de plus nombreuses récurrences et s'interroger sur leur signification. Les travaux de Sigrid Schmidt fournissent de bien plus riches aperçus sur les interactions entre les folklores san et afrikaner ${ }^{2}$.

Restent les contes eux-mêmes, qui appartiennent à la très riche collection de l'auteur, constituée pour l'essentiel dans les années 1960 et 1970 lors de voyages d'étude à travers toute l'Afrique australe. L'ouvrage en présente vingtcinq provenant d'Afrique du Sud et de ses enclaves (Lesotho et Swaziland), qui sont en général d'une grande qualité, dénotant parfois un formidable souffle dramatique. On pourra regretter le manque de précision sur le statut des textes dans la population (xhosa, zulu, ndebele, swazi, san, sotho) où ils ont été recueillis, ou encore sur leur fréquence dans la littérature orale : dans quelle mesure s'agit-il de contes «traditionnels », de créations, de mythes ? Certes, ce silence procède sans doute d'un parti pris qui vise à ignorer la question du statut pour mieux intégrer la littérature orale africaine dans un projet global de réflexion sur la création littéraire (un peu comme l'exposition du Louvre sur les arts premiers abolit la question du contexte de production des oeuvres plastiques pour les insérer dans une démarche esthétique dont elles étaient auparavant exclues). Mais alors, pourquoi rester au milieu du gué, présentant d'un côté chacune des conteuses comme autant d'« auteurs » (à égalité avec Pauline Smith), et indiquant de l'autre son ethnie au-dessous du titre (mais pas pour Pauline Smith) ? Quoi qu'il en soit, il nous semble qu'une oeuvre de l'esprit gagne toujours à être resituée dans un contexte de production, ce que paraît refuser Harold Scheub (hormis par ses quelques allusions aux incontournables « rites de passage »). Mais il est vrai que ce n'est pas ici son propos. Les personnes intéressées par l'Afrique du Sud découvriront cependant dans ces contes des univers mentaux marqués par une inventivité ancrée dans les terroirs sud-africains, mais aussi profondément touchés par un contexte social qui est celui de l'apartheid : nombre de situations naissent de la recherche d'un travail, de l'habitude des déplacements à longue distance (vers le lieu de 
travail, l'école, la famille...), manifestant la récurrence de la violence domestique ou sociale ainsi que la présence du Blanc (parfois présenté comme l'ogre) à l'horizon de l'espace vécu.

\section{NOTES}

1. Sur //Kabbo, et plus généralement sur le fonds Bleek et Lloyd, voir Janette Deacon \& Thomas A. Dowson, eds, Voices from the Past. /Xam Bushmen and the Bleek and Lloyd Collection, Johannesburg, Witwatersrand University Press, 1996. Recension : FrançoisXavier Fauvelle-Aymar, «Quel passé pour les Khoisan ? Représentations, mémoire, héritages ", Cahiers d'Études africaines, 1999, XXXIX (3-4), 155-156 : 979-985, passim. 2. Voir par exemple Sigrid Schmidt, « Tales and Beliefs about Eyes-on-his-feet : the Interrelatedness of Khoisan Folklore », in M. Biesele, ed., The Past and Future of !Kung Ethnography, Hambourg, H. Buske, 1986 : 169-194. 